\title{
Global Exponential Stability of Impulsive Cohen-Grossberg-Type BAM Neural Networks with Time-Varying and Distributed Delays
}

\author{
Haydar Akça, Jamal Benbourenane, and Valéry Covachev
}

\begin{abstract}
The purpose of this paper is to investigate the global exponential stability of a class of impulsive bidirectional associative memories (BAM) neural networks that possesses Cohen-Grossberg dynamics. By constructing and using some inequality techniques and a fixed point theorem sufficient conditions are obtained to ensure the existence and global exponential stability of the solutions for impulsive Cohen-Grossberg neural networks with time delays and distributed delays.
\end{abstract}

Index Terms-Cohen-grossberg neural networks, impulses, globally exponential stability, time delay, distributed delay.

\section{INTRODUCTION}

The Cohen-Grossberg neural network models proposed by Cohen and Grossberg [1] have been widely applied to various problems in scientific and engineering fields [1]. BAM neural networks are useful in many fields such as pattern recognition and automatic control, the stability properties and applications of BAM's models have been researched by many scholars see [2]-[6] and references are given therein. Most widely used neural networks are neither of purely continuous-time nor of purely discrete-time type [7]-[10]. Also there has been a new category of neural networks called impulsive neural networks, which display a combination of characteristics of both continuous-time and discrete-time systems. The bidirectional associative memory (BAM) neural network model known as an extension of the unidirectional auto-associator of Hopfield [11], and references cited therein. The method was introduced first by Kosko [12].

To the best of our knowledge, there are few results on the stability of impulsive Cohen-Grossberg neural networks with both time-varying and distributed delays [9]-[10]. We study the stability problem of BAM impulsive Cohen-Grossberg neural networks with time-varying and distributed delays and derived to guarantee the global asymptotic stability of the solution by using some inequality techniques, fixed point theorem and some analysis techniques. We consider impulsive Cohen-Grossberg-type BAM neural networks with time-varying and distributed delays which are described by

Manuscript received February 12, 2014; revised April 28, 2014.

H. Akça and J. Benbourenane are with the Applied Sciences and Mathematics Department, College of Arts and Sciences, Abu Dhabi University, P.O. Box 59911, Abu Dhabi, UAE (e-mail: Haydar.Akca@adu.ac.ae, Jamal.Benbourenane@adu.ac.ae).

V. Covachev is with the Bulgarian Academy of Sciences, Sofia, Bulgaria (e-mail: covachev@hotmail.com). the following functional integro-differential equations:

$$
\begin{aligned}
& \int \frac{d x_{i}(t)}{d t}=a_{i}\left(x_{i}(t)\right)\left[-\alpha_{i}\left(x_{i}(t)\right)+\sum_{j=1}^{m} p_{j i} f_{j}\left(y_{j}\left(t-\tau_{j i}(t)\right)\right)\right. \\
& \left.+\sum_{j=1}^{m} \int_{0}^{\infty} r_{j i}(s) f_{j}\left(y_{j}(t-s)\right) d s+I_{i}\right] \\
& t>0, \quad t \neq t_{k}, \quad i=1,2, \ldots, n, \\
& \Delta x_{i}\left(t_{k}\right)=x_{i}\left(t_{k}^{+}\right)-x_{i}\left(t_{k}^{-}\right)=\bar{I}_{k}\left(x_{i}\left(t_{k}\right)\right) \text {, } \\
& i=1,2, \ldots, n, k=1,2, \ldots, \\
& \frac{d y_{j}(t)}{d t}=b_{j}\left(y_{j}(t)\right)\left[-\beta_{j}\left(y_{j}(t)\right)+\sum_{i=1}^{n} q_{i j} g_{i}\left(x_{i}\left(t-\sigma_{i j}(t)\right)\right)\right. \\
& \left.+\sum_{i=1}^{n} \int_{0}^{\infty} s_{i j}(s) g_{i}\left(x_{i}(t-s)\right) d s+J_{j}\right] \\
& t>0, t \neq t_{k}, j=1,2, \ldots, m, \\
& \Delta y_{j}\left(t_{k}\right)=y_{j}\left(t_{k}^{+}\right)-y_{j}\left(t_{k}^{-}\right)=\bar{J}_{k}\left(y_{j}\left(t_{k}\right)\right) \text {, } \\
& j=1,2, \ldots, m, k=1,2, \ldots, \\
& \begin{cases}x_{i}(s)=\varphi(s), & s \in(-\infty, 0], \quad i=1,2, \ldots, n, \\
y_{j}(s)=\phi(s), & s \in(-\infty, 0], j=1,2, \ldots, m,\end{cases}
\end{aligned}
$$

where $n$ and $m$ correspond to the number of neurons in the $X$ - layer and $Y$-layer, respectively, $\varphi(s)$ and $\phi(s)$ are bounded continuous functions on $(-\infty, 0]$, and $\Delta x_{i}\left(t_{k}\right)=x_{i}\left(t_{k}^{+}\right)-x_{i}\left(t_{k}^{-}\right), \Delta y_{j}\left(t_{k}\right)=y_{j}\left(t_{k}^{+}\right)-y_{j}\left(t_{k}^{-}\right)$are the impulses at the moments $t_{k}$ and $0<t_{1}<t_{2}<\cdots$ is a strictly increasing sequence such that $\lim _{t \rightarrow \infty} t_{k}=+\infty$, and $x_{i}(t), y_{j}(t)$ are the activations of the $i$ th neuron in $F_{X}$ and the $j$ th neuron in $F_{Y}$, respectively. The functions $a_{i}, b_{j}$ are abstract amplification functions and $\alpha_{i}, \beta_{j}$ are self-excitation rate functions. $\tau_{j i}(t), \sigma_{i j}(t)$ are positive time delays corresponding to the finite speed of the axonal signal transmission. $f_{j}, g_{i}$ present the activation functions of the neuron. The functions $p_{j i}, q_{i j}, r_{j i}(t), s_{i j}(t)$ are the connection weights, they denote the strengths of connectivity between the cell $j$ in $F_{Y}$ and cell $i$ in $F_{X}$ at time $t, t-\tau_{j i}(t), t-\sigma_{i j}(t)$, and $I_{i}, J_{j}$ denote the $i$ th and $j$ th component of an external input source introduced from 
outside the network to the cell $i$ in $F_{X}$ and the cell $j$ in $F_{Y}$, respectively (see [8] and references cited therein). As usual in the theory of impulsive differential equations, at the points of discontinuity $t_{k}$ of the solution

$$
t \mapsto\left(x_{1}(t), x_{2}(t), \ldots, x_{n}(t), y_{1}(t), y_{2}(t), \ldots, y_{m}(t)\right)^{T}
$$

We assume that

$$
\begin{aligned}
& x_{i}\left(t_{k}\right)=x_{i}\left(t_{k}-0\right), y_{j}\left(t_{k}\right)=y_{j}\left(t_{k}-0\right), \\
& x_{i}^{\prime}\left(t_{k}\right)=x_{i}^{\prime}\left(t_{k}-0\right), y_{j}^{\prime}\left(t_{k}\right)=y_{j}^{\prime}\left(t_{k}-0\right),
\end{aligned}
$$

where $i=1,2, \ldots, n, j=1,2, \ldots, m$. The vector function

$$
\left(x_{1}(t), x_{2}(t), \ldots, x_{n}(t), y_{1}(t), y_{2}(t), \ldots, y_{m}(t)\right)^{T}
$$

is said to be a solution of the system (1)-(1)(2).

Throughout this paper we assume the following conditions are satisfied:

H1: $a_{i}\left(x_{i}\right)>0, b_{j}\left(y_{j}\right)>0$ and $a_{i}, b_{j}$ are bounded, that is, there exist positive constants $\underline{a}_{i}, \bar{a}_{i}$, and $\underline{b}_{j}, \bar{b}_{j}$ such that

$$
\begin{aligned}
& \underline{a}_{i} \leq a_{i}\left(x_{i}\right) \leq \bar{a}_{i}, \quad i=1,2, \ldots, n, \\
& \bar{a}=\max \left\{\bar{a}_{i}\right\}, \quad \underline{a}=\min \left\{\underline{a}_{i}\right\}, \\
& \underline{b}_{j} \leq b_{j}\left(y_{j}\right) \leq \bar{b}_{j}, \quad j=1,2, \ldots, m, \\
& \bar{b}=\max \left\{\bar{b}_{j}\right\}, \quad \underline{b}=\min \left\{\underline{b}_{j}\right\}, \\
& a=\max \{\bar{a}, \bar{b}\}, \quad b=\min \{\underline{a}, \underline{b}\} .
\end{aligned}
$$

H2: The delayed feedback functions $r_{j i}, s_{i j}$ are real-valued nonnegative continuous functions defined on $[0, \infty)$ with

$$
\int_{0}^{\infty} r_{j i}(s) d s \leq r_{j i}, \quad \int_{0}^{\infty} s_{i j}(s) d s \leq s_{i j} .
$$

Here $r_{j i}, s_{i j}$ are nonnegative constants. There exists a positive constant number, such that

$$
\int_{0}^{\infty} e^{\varepsilon s} r_{j i}(s) d s<\infty, \int_{0}^{\infty} e^{\varepsilon s} s_{i j}(s) d s<\infty
$$

and there must be constants $\lambda_{1}, \lambda_{2}$ satisfying

$$
\begin{aligned}
& \left\|\int_{0}^{\infty} R(s) d s\right\|_{2} \leq\left\|\int_{0}^{\infty} e^{\varepsilon s} R(s) d s\right\|_{2}=\lambda_{1}, \\
& \left\|\int_{0}^{\infty} S(s) d s\right\|_{2} \leq\left\|\int_{0}^{\infty} e^{\varepsilon s} S(s) d s\right\|_{2}=\lambda_{2},
\end{aligned}
$$

where $R(s)$ and $S(s)$ are matrix-valued functions with entries respectively $r_{j i}(s)$ and $s_{i j}(s)$ (see below).

H3: There exist positive constant numbers $L_{j}, M_{i}$ such that

$$
\begin{aligned}
& \left|f_{j}(u)-f_{j}(v)\right| \leq L_{j}|u-v|, \quad j=1,2, \ldots, m, \\
& \left|g_{i}(u)-g_{i}(v)\right| \leq M_{i}|u-v|, \quad i=1,2, \ldots, n,
\end{aligned}
$$

for any $u, v \in \mathbb{R}$ and there exist positive constants $\gamma_{i}, \xi_{j}$ such that

$$
\frac{\alpha_{i}(u)-\alpha_{i}(v)}{u-v} \geq \gamma_{i}>0, \quad \frac{\beta_{j}(u)-\beta_{j}(v)}{u-v} \geq \xi_{j}>0
$$

for any $u, v \in \mathbb{R}, i=1,2, \ldots, n, j=1,2, \ldots, m$ and $u \neq v$.

H4: The impulsive operators

$$
\begin{gathered}
\bar{I}_{k}\left(x\left(t_{k}\right)\right), \bar{J}_{k}\left(y\left(t_{k}\right)\right) \text { satisfy [10] } \\
\bar{I}_{k}\left(x_{i}\left(t_{k}\right)\right)=-l_{1}\left(x_{i}\left(t_{k}\right)-x_{i}^{*}\right), \quad 0<l_{1}<2, i=1,2, \ldots, n, \\
\bar{J}_{k}\left(y_{j}\left(t_{k}\right)\right)=-l_{2}\left(y_{j}\left(t_{k}\right)-y_{j}^{*}\right), \quad 0<l_{2}<2, j=1,2, \ldots, m .
\end{gathered}
$$

For the sake of convenience, we can rewrite system (1) in the form

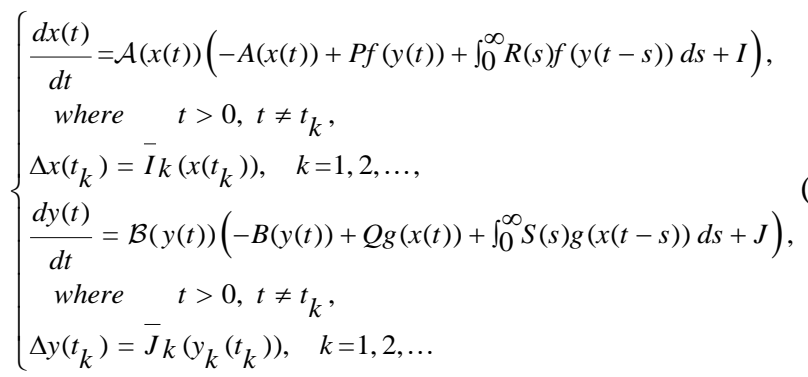

where

$$
\begin{gathered}
x(t)=\operatorname{col}\left\{x_{i}(t)\right\}, \quad y(t)=\operatorname{col}\left\{y_{j}(t)\right\}, \\
\mathcal{A}(x)=\operatorname{diag}\left\{a_{i}\left(x_{i}\right)\right\}, \\
\mathcal{B}(y)=\operatorname{diag}\left\{b_{j}\left(y_{j}\right)\right\}, \quad A(x)=\operatorname{diag}\left\{\alpha_{i}\left(x_{i}\right)\right\}, \\
B(y)=\operatorname{diag}\left\{\beta_{j}\left(y_{j}\right)\right\},
\end{gathered}
$$

$$
\begin{aligned}
& P=\left(p_{j i}\right)_{m \times n}, \quad R(s)=\left(r_{j i}(s)\right)_{m \times n}, \\
& f(t)=\operatorname{col}\left\{f_{j}(t)\right\}, \quad I=\operatorname{col}\left\{I_{i}\right\}, \\
& Q=\left(q_{i j}\right)_{n \times m}, \quad S(s)=\left(s_{i j}(s)\right)_{n \times m}, \\
& g(t)=\operatorname{col}\left\{g_{i}(t)\right\}, \quad J=\operatorname{col}\left\{J_{j}\right\} .
\end{aligned}
$$

Assume that system (3) has a unique equilibrium point $\left(x^{*}, y^{*}\right)$, then let

$$
\begin{gathered}
u(t)=x(t)-x^{*}, v(t)=y(t)-y^{*}, \quad \widetilde{\mathcal{A}}(u)=\mathcal{A}\left(u+x^{*}\right), \\
\widetilde{\mathcal{B}}\left(v=\mathcal{B}\left(v+y^{*}\right),\right. \\
\tilde{A}(u)=A\left(u+x^{*}\right)-A\left(x^{*}\right), \quad \tilde{B}(v)=B\left(v+y^{*}\right)-B\left(y^{*}\right), \\
\tilde{f}(v)=f\left(v+y^{*}\right)-f\left(y^{*}\right), \\
\tilde{g}(u)=g\left(u+x^{*}\right)-g\left(x^{*}\right), \\
\Psi(t)=\varphi(t)-x^{*}, \Phi(t)=\phi(t)-y^{*},
\end{gathered}
$$

then system (3) can be written as 


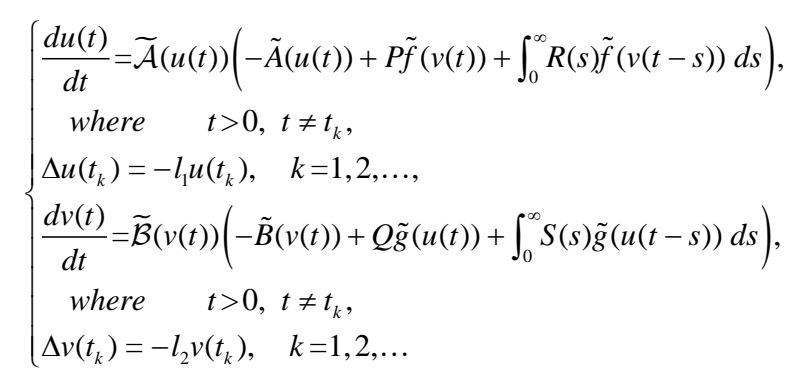

The initial conditions associated with system (4) can be defined as

$$
u(s)=\Psi(s), \quad v(s)=\Phi(s), \quad s \in(-\infty, 0] .
$$

For

$$
u=\left(u_{1}, u_{2}, \ldots, u_{n+m}\right)^{T} \in \mathbb{R}^{m+n}, \phi:(-\infty, 0] \rightarrow \mathbb{R}^{m+n}
$$

we define norms by

$$
\begin{aligned}
& \|u\|=\sum_{k=1}^{n+m}\left|u_{k}\right|, \\
& \|\phi\|=\sup _{s \in(-\infty, 0]}\|\phi(s)\| .
\end{aligned}
$$

Let us denote

$$
\tau=\max _{1 \leq i \leq n, 1 \leq j \leq m}\left\{\tau_{j i}(t), \sigma_{i j}(t)\right\}
$$

and let $\varphi_{i}(\cdot), \psi_{j}(\cdot)$ be real-valued continuous functions defined on $(-\infty, 0]$. The model introduced $(1)-(1)(2)$ is studied [7], [8], [10] with delays and impulses.

\section{MAIN RESUlts}

If $\left(x_{1}^{*}, x_{2}^{*}, \ldots x_{n}^{*}, y_{1}^{*}, y_{2}, \ldots, y_{m}^{*}\right)^{T}$ is an equilibrium point of system (1), then it satisfies

$$
\left\{\begin{array}{l}
A\left(x^{*}\right)=P f\left(y^{*}\right)+\int_{0}^{\infty} R(s) d s f\left(y^{*}\right)+I, \\
B\left(y^{*}\right)=Q g\left(x^{*}\right)+\int_{0}^{\infty} S(s) d s g\left(x^{*}\right)+J,
\end{array}\right.
$$

where the impulses $\bar{I}_{k}(\cdot), \bar{J}_{k}(\cdot)$ satisfy

$$
\bar{I}_{k}\left(x^{*}\right)=0, \bar{J}_{k}\left(y^{*}\right)=0 \text {. }
$$

The equilibrium point $\left(x^{*}, y^{*}\right)$ of the system (3) is said to be globally exponentially stable, if there exist constants $\varepsilon>0$ and $E \geq 1$ such that

$$
\left\|x(t)-x^{*}\right\|_{2}+\left\|y(t)-y^{*}\right\|_{2} \leq E\left(\left\|\varphi-x^{*}\right\|+\left\|\phi-y^{*}\right\|\right) e^{-\varepsilon t}
$$

for all $t>0$, and $x(t), y(t)$ is any solution of the system (3) with initial values $(\varphi(s), \phi(s))$.

Theorem 1 [10] Under the assumptions $H 1, H 2$ and $H 3$ the solutions of the system (4) are uniformly bounded.

Proof. For $t \neq t_{k}$, on the first partition interval $0<t<t_{1}$ there exists a sufficiently large number $M$ such that if

$$
\|\Psi\|<\frac{M}{2}, \quad\|\Phi\|<\frac{M}{2},
$$

then

$$
\|u(t)\|+\|v(t)\|<M
$$

Then without loss of generality, integrating system (4) on the interval $\left[0, t_{1}\right]$ and using the assumptions $H 1-H 4$ we get

$$
\begin{aligned}
&\left\|u\left(t_{1}\right)\right\|+\left\|v\left(t_{1}\right)\right\| \leq e^{-a_{0} t_{1}}\|\Psi\| \\
&+\int_{0}^{t_{1}} e^{-a_{0}\left(t_{1}-\theta\right)}\|P\| \xi\|v(\theta)\| d \theta \\
&+\int_{0}^{t_{1}} e^{-a_{0}\left(t_{1}-\theta\right)} \xi\left\|\int_{0}^{\infty} R(s) v(\theta-s)\right\| d \theta
\end{aligned}
$$

$$
\begin{array}{r}
+e^{-b_{0} t_{1}}\|\Phi\|+\int_{0}^{t_{1}} e^{-b_{0}\left(t_{1}-\theta\right)}\|Q\| \eta \| u(\theta \| d \theta \\
+\int_{0}^{t_{1}} e^{-b_{0}\left(t_{1}-\theta\right)} \eta\left\|\int_{0}^{\infty} S(s) u(\theta-s)\right\| d \theta \\
\leq e^{-a_{0} t_{1}} \frac{M}{2}+\int_{0}^{t_{1}} e^{-a_{0}\left(t_{1}-\theta\right)}\|P\| \xi \frac{M}{2} d \theta \\
+\int_{0}^{t_{1}} e^{-a_{0}\left(t_{1}-\theta\right)} \xi\left\|\int_{0}^{\infty} e^{\varepsilon s} R(s) d s\right\| \frac{M}{2} d \theta \\
+e^{-b_{0} t_{1}} \frac{M}{2}+\int_{0}^{t_{1}} e^{\left.-b_{0} t_{1}-\theta\right)}\|Q\| \eta \frac{M}{2} d \theta \\
+\int_{0}^{t_{1}} e^{-b_{0}\left(t_{1}-\theta\right)} \eta\left\|\int_{0}^{\infty} e^{\varepsilon s} S(s) d s\right\| \frac{M}{2} d \theta
\end{array}
$$

Let

$$
\max \left\{\lambda_{1}, \lambda_{2}\right\}=\lambda, \max \{\|P\|,\|Q\|\}=\Gamma, \max \{\xi, \eta\}=\zeta
$$

and $a_{0}, b_{0}>1$ and $\Xi>0$ is a positive constant with the assumptions $H 1-H 4$ and after simplification of the system (6), we get

$$
\begin{aligned}
& \left\|u\left(t_{1}\right)\right\|+\left\|v\left(t_{1}\right)\right\| \leq \\
& e^{-a_{0} t_{1}} \frac{M}{2}+\left[\frac{1-e^{-a_{0} t_{1}}}{a_{0}}+\frac{1-e^{-a_{0} t_{1}}}{a_{0}}\right] \Gamma \lambda \zeta \frac{M}{2} \\
& +e^{-b_{0} t_{1}} \frac{M}{2}+\left[\frac{1-e^{-b_{0} t_{1}}}{b_{0}}+\frac{1-e^{-b_{0} t_{1}}}{b_{0}}\right] \Gamma \lambda \zeta \frac{M}{2} \\
& =\left[\frac{e^{-a_{0} t_{1}}+e^{-b_{0} t_{1}}}{2}+\left(\frac{1-e^{-a_{0} t_{1}}}{a_{0}}+\frac{1-e^{-b_{0} t_{1}}}{b_{0}}\right) \Gamma \lambda \zeta\right] M \\
& =\Gamma \lambda \zeta \frac{M}{2}\left[e^{-a_{0} t_{1}}+e^{-b_{0} t_{1}}+1-e^{-a_{0} t_{1}}+1-e^{-b_{0} t_{1}}\right] \\
& =\Gamma \lambda \zeta M=\Xi
\end{aligned}
$$


Repeating the above procedure on the successive interval we can easily conclude that the solutions of the system (4) are uniformly bounded. The proof is completed.

Theorem 2. [10] Assume that $H 1-H 4$ are satisfied, then the system (3) has a unique equilibrium point which is a solution of the system (5).

Proof. We proved in Theorem 1 that all solutions of system (4) are bounded, that means for any initial value, if

$$
\bar{I}_{k}\left(x_{i}^{*}\right)=0, \bar{J}_{k}\left(y_{j}^{*}\right)=0, i=1,2, \ldots, n, \quad j=1,2, \ldots, m,
$$

then system (3) has a unique equilibrium point. Then we have

$$
\begin{aligned}
& \left\|P f(y(t))+\int_{0}^{\infty} R(s) f(y(t-s)) d s+I\right\| \\
& \leq\|P\| \xi\|y(t)\|+\xi\left\|\int_{0}^{\infty} R(s) y(t-s) d s\right\| \\
& +\|I\| \\
& <\|P\| \xi\|y(t)\|+\xi\|y(t)\| \lambda_{1}+\|I\| \\
& <\|y(t)\|(\Gamma \zeta+\zeta \lambda)+\|I\| \\
& \leq \pi_{1} .
\end{aligned}
$$

Similarly we can have

$$
\begin{aligned}
& \left\|Q g(x(t))+\int_{0}^{\infty} S(s) g(x(t-s)) d s+J\right\| \\
& \leq\|Q\| \eta\|x(t)\|+\eta\left\|\int_{0}^{\infty} S(s) x(t-s) d s\right\|+\|J\| \\
& <\|Q\| \eta\|x(t)\|+\eta\|x(t)\| \lambda_{2}+\|J\| \\
& <\|x(t)\|(\Gamma \zeta+\zeta \lambda)+\|J\| \leq \pi_{2} .
\end{aligned}
$$

Let us define $T(t)=A(x(t))$ and $W(t)=B(y(t))$ in such a way that

$$
\begin{aligned}
& T(t)=P f\left(B^{-1}(W(t))\right)+\int_{0}^{\infty} R(\tau) f\left(B^{-1}(W(\tau))\right) d \tau+I, \\
& W(t)=Q g\left(A^{-1}(T(t))\right)+\int_{0}^{\infty} S(\tau) g\left(A^{-1}(T(\tau))\right) d \tau+J .
\end{aligned}
$$

The systems of inequalities (7)-(7)(8) suggests us to define a set $\Omega \subset \mathbb{R}^{n+m}$ by

$$
\Omega=\left\{(T, W), \text { such that }\|T\| \leq \pi_{1},\|W\| \leq \pi_{2}\right\} .
$$

If $(T, W),(\bar{T}, \bar{W})$ are any two different points of $\Omega$, then

$$
\begin{aligned}
& \|F(T, W)-F(\bar{T}, \bar{W})\| \\
& =\| P\left(f\left(B^{-1}(W(t))\right)-f\left(B^{-1}(\bar{W}(t))\right)\right)
\end{aligned}
$$$$
+\int_{0}^{\infty} R(\tau) f\left(B^{-1}(W(\tau))\right) d \tau
$$$$
-\int_{0}^{\infty} R(\tau) f\left(B^{-1}(\bar{W}(\tau))\right) d \tau \|
$$$$
+\| Q\left(g\left(A^{-1}(T(t))\right)-Q g\left(A^{-1}(\bar{T}(t))\right)\right)
$$

$$
\begin{aligned}
& +\int_{0}^{\infty} S(\tau) g\left(A^{-1}(T(\tau))\right) d \tau \\
& -\int_{0}^{\infty} S(\tau) g\left(A^{-1} \bar{T}(\tau)\right) d \tau \| \\
& \quad \leq\|P\|\left\|f\left(B^{-1}(W(t))\right)-f\left(B^{-1}(\bar{W}(t))\right)\right\| \\
& +\left\|\int_{0}^{\infty} R(\tau)\left(f\left(B^{-1}(\bar{W}(\tau))\right)-f\left(B^{-1}(W(\tau))\right)\right) d \tau\right\| \\
& +\|Q\|\left\|g\left(A^{-1}(T(t))\right)-g\left(A^{-1}(\bar{T}(t))\right)\right\|
\end{aligned}
$$

$$
+\left\|\int_{0}^{\infty} S(\tau)\left(g\left(A^{-1}(\bar{T}(\tau))\right)-g\left(A^{-1}(T(\tau))\right)\right) d \tau\right\| .
$$

Using the inequalities (7)-(7)(8) it can be easily concluded that

$$
\begin{aligned}
& \|F(T, W)-F(\bar{T}, \bar{W})\| \\
& \leq \Gamma \zeta\left\|B^{-1}\right\|\|W(t)-\bar{W}(t)\|+\lambda \zeta\left\|B^{-1}\right\|\|\bar{W}(t)-W(t)\| \\
& +\Gamma \zeta\left\|A^{-1}\right\|\|T(t)-\bar{T}(t)\| \lambda \zeta\left\|A^{-1}\right\|\|\bar{T}(t)-T(t)\| \\
& =\zeta(\Gamma-\lambda)\left(\left\|B^{-1}\right\|\|W(t)-\bar{W}(t)\|+\left\|A^{-1}\right\|\|T(t)-\bar{T}(t)\|\right) \\
& \leq \gamma(\|W(t)-\bar{W}(t)\|+\|T(t)-\bar{T}(t)\|)=\gamma\|(W, T)-(\bar{W}, \bar{T})\| \\
& \text { Provided that } 0<\gamma<1 \text { where }
\end{aligned}
$$

$$
\gamma=\max \left\{\zeta(\Gamma-\lambda)\left\|B^{-1}\right\|, \zeta(\Gamma-\lambda)\left\|A^{-1}\right\|\right\} .
$$

Because of the contraction mapping principle the mapping $F$ has a unique fixed point $\left(W^{*}, T^{*}\right)$ and this result completes the proof.

Theorem 3. Suppose that the assumptions $H 1-H 4$ are satisfied, then the equilibrium point of the system (4) is globally exponentially stable.

Proof. From $H 1-H 2$ define $0<\varepsilon<a$ and

$$
\Theta=\max \left\{\frac{\zeta(\Gamma-\lambda)\left\|B^{-1}\right\|}{a-\varepsilon}, \frac{\zeta(\Gamma-\lambda)\left\|A^{-1}\right\|}{a-\varepsilon}\right\}<1
$$


To prove the assertion for $t \neq t_{k}$ for any $\Psi, \Phi \in C$ and $\beta>1$ it can be shown that the following inequality holds:

$$
\|u(t)\|+\|v(t)\|<\beta E(\|\Psi\|+\|\Phi\|) e^{-\varepsilon t}
$$

for all $t>0$ and $t \neq t_{k}$

Assume that (13) is not true and there must be some $t_{2}>0$ such that

$$
\left\|u\left(t_{2}\right)\right\|+\left\|v\left(t_{2}\right)\right\|=\beta E(\|\Psi\|+\|\Phi\|) e^{-\varepsilon t_{2}}
$$

for $t_{2}>0$, and $t_{2} \neq t_{k}$.

Following the similar procedure presented in the reference [10], one can easily reach a contradiction and that result completes the proof of the theorem.

\section{CONCLUSION}

We study the stability problem of BAM impulsive Cohen-Grossberg neural networks with time-varying and distributed delays by constructing and using some inequality techniques and fixed point theorem. We obtained sufficient conditions to ensure the existence and global exponential stability of the solutions for impulsive Cohen-Grossberg neural networks with time-varying and distributed delays.

\section{REFERENCES}

[1] M. A. Cohen and S. Grossberg, "Absolute stability of global pattern formation and parallel memory storage by competitive neural networks," IEEE Trans. Systems Man Cybern, vol. 13, no. 5, pp. 815-826, 1983.

[2] H. Akça and V. Covachev, "Impulsive Cohen-Grossberg neural networks with S-type distributed delays," Tatra Mt. Math. Publ., vol. 48, pp. 1-13, Dec. 2011.

[3] H. Akça and V. Covachev, "Spatial discretization of an impulsive Cohen-Grossberg neural network with time-varying and distributed delays and reaction-diffusion terms," An. Stiint Univ. Ovidius Constanta Ser. Mat., vol. 17, no. 3, pp. 15-26, 2009.

[4] V. Covachev, H. Akça, and S. Makhtar, "Discrete-time counterparts of impulsive Cohen-Grossberg neural networks of neutral type," Neural Parallel Sci. Comput., vol. 19, no. 3/4, pp. 345-360, 2011.

[5] S. Mohamad, H. Akça, and V. Covachev, "Discrete-time analogues of impulsive Cohen-Grossberg neural networks with transmission delays," Int. J. Math. Comput., vol. 1, pp. 124-143, November 2008.

[6] S. Mohamad, H. Akça, and V. Covachev, "Discrete-time Cohen-Grossberg neural networks with transmission delays and impulses," Tatra Mt. Math. Publ., vol. 43, pp. 145-161, Dec. 2009.

[7] C. Bai, "Stability analysis of Cohen-Grossberg BAM neural networks with delays and impulses," Chaos, Solitons and Fractals, vol. 35, pp. 263-267, Jan. 2008.

[8] F. Yang, C. Zhang, and D. Wu, "Global stability analysis of impulsive BAM type Cohen-Grossberg neural networks with delays," Appl. Math. Comput., vol. 186, pp. 932-940, March 2007.
[9] Y. Li and C. Yang, "Global exponential stability analysis on impulsive BAM neural networks with distributed delays," J. Math. Anal. Appl., vol. 324, pp. 1125-1139, 15 Dec. 2006

[10] J. Zhou and S. Li, "Global exponenetial stability of impulsive BAM neural networks with distributed delays," Neurocomputing, vol. 72, pp. 1688-1693, March 2009.

[11] J. J. Hopfield, "Neuron with graded response have collective computational properties like those of two-state neurons," in Proc. Nat. Acad. Sci., USA, 1984, pp. 3088-3092.

[12] B. Kosko, "Bi-directional associative memories," IEEE Trans. Systems Man Cybern., vol. 18, no. 1, pp. 49-60, 1988.

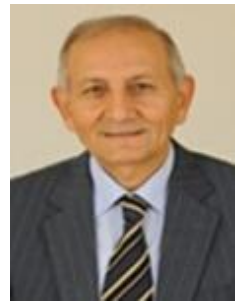

Haydar Akca graduated in mathematics and astronomy from Ege University, Faculty of Science, in 1970. Dr. Akca received Ph. D. in applied mathematics from Inonu University, Malatya with collaboration Helsinki University of Technology in 1983. Since then, he has been teaching in various universities. He becomes a professor in applied mathematics at Akdeniz University, Antalya in 1996. He has around 95 technical publications including two monographs, and his research interest area includes primarily functional differential equations, neural networks, mathematical modeling, control theory, and wavelet neural networks. He has been organizing the serial international conference on dynamical systems and applications. Dr. Akca is a member of a number of professional mathematical associations. He is the editor-in-chief and editorial board member of number of international mathematical journals. At present he is a professor of applied mathematics at the Abu Dhabi University, College of Arts and Science Department of Applied Sciences and Mathematics, Abu Dhabi, United Arab Emirates.

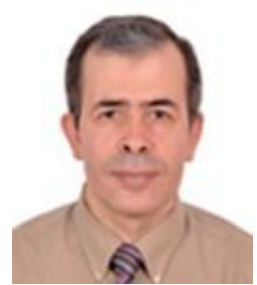

Jamal Benbourenane is an assistant professor at the applied science and mathematics, College of Art and Science at Abu Dhabi University, United Arab Emirates. He graduated in 2001 with a $\mathrm{PhD}$ degree and a master degree in mathematics from Northern Illinois University, IL, USA and a bachelor in probability \& statistics from Algiers. He taught at Indiana University at South Bend, IN, Valdosta State University, GA, in USA and at United Arab Emirates University before joining Abu Dhabi University in UAE.

Dr. Jamal has a very strong background in mathematics and statistics and a long experience in teaching mathematics at different levels with different types of new technologies. His research interests are in complex analysis and complex differential equations as well as real analysis, functional differential equations and neural networks.

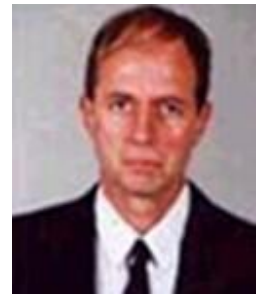

Valéry Covachev graduated in mathematics from Sofia University, Faculty of Mathematics, in 1980 Dr. Covachev obtained Ph.D. in applied mathematics from Belarus State University, Minsk, in 1985. Since then, he has been teaching in various universities. He became an associate professor in differential equations at the Institute of Mathematics, Bulgarian Academy of Sciences, Sofia in 1993. He has about 100 publications including two monographs, and his research interest area includes primarily functional and impulsive differential equations and neural networks. He has been participating in the organization of several Colloquia in differential equations and numerical analysis in Plovdiv, Bulgaria, 1990-1995, and the serial international conference on dynamical systems and applications. Dr. Covachev is a member of a number of professional associations such as SIAM and IEEE. He is an editorial board member of a number of international mathematical journals. At present he is an associate professor of applied mathematics at Department of Mathematics and Statistics, College of Science, Sultan Qaboos University, Muscat, Sultanate of Oman. 